\title{
Explaining to different audiences the new definition and experimental realizations of the kilogram
}

\author{
Joaquín Valdés \\ Universidad Nacional de San Martín, 25 de Mayo y Francia, 1650 San Martín, Prov. Buenos Aires, Argentina \\ Correspondence: Joaquín Valdés (jovaldes1875@gmail.com)
}

Received: 29 September 2020 - Revised: 23 November 2020 - Accepted: 30 November 2020 - Published: 21 January 2021

\begin{abstract}
Different options were discussed before reaching the final agreement on the new definitions of the SI units, effective from 20 May 2019, especially with regard to the kilogram, now defined in terms of the numerical value of the Planck constant $(h)$. Replacing the artefact definition of the kilogram with a new one based on the mass of a particle, or the atomic mass constant $\left(m_{u}\right)$, would have been preferable for ease of understanding, among other reasons. In this paper we discuss some limitations of teaching to different audiences what a kilogram is in the redefined International System of Units (SI), including realizations of the new definition.
\end{abstract}

\section{Understanding a definition of the kilogram based on the Planck constant}

On 16 November 2018, the General Conference of Weights and Measures - CGPM (from the French Conférence Générale des Poids et Mesures) decided that, effective from 20 May 2019, the International System of Units, the SI, is the system of units defined in terms of seven defining constants. This is the result of three decades of progress in metrology (Stock et al., 2019). It allows the dream of realizing the units at any time and place. The new definition of the kilogram fixing the value of the Planck constant abolished the previous one, which was referred to as an artefact. It allows the realization of mass at any scale using different technologies. The kilogram is no longer the mass of "the weight that was in Paris", but it is defined by taking the fixed numerical value of $h$ to be exactly $6.62607015 \times 10^{-34}$ when expressed in the unit joules per second. Now we have to explain what this new kilogram is to wider audiences, including technicians and university graduates from the most diverse disciplines, who do not necessarily know what the Planck constant $h$ is. This difficulty was already known from the very beginning of discussions around the redefinition of the SI. The question of understandability was present in several important publications, at least since 2007, until shortly before the final approval of the redefinition (Becker et al., 2007; Fletcher et al., 2015). One prevailing argument to support the definition of the kilogram based on a fixed value of $h$ was the need for electrical metrology to bring into the SI the realizations of the volt and the ohm, providing exact numerical values for the Josephson constant $K_{\mathrm{J}}=2 e / h$ and the von Klitzing constant $R_{\mathrm{K}}=h / e^{2}$. The second argument was that $h$ is a constant more fundamental than the mass of an elementary particle.

\subsection{School level}

An evasive way to explain what a kilogram now is, avoiding mentioning the Planck constant, would be not to answer exactly what the new definition says, explaining instead that one of the possible ways of "realizing" the new kilogram is by counting atoms. Most people may have some idea of what an atom is, so they can imagine that by putting together a huge number of atoms one obtains a mass similar to that of the old platinum iridium artefact that served as an international prototype of the kilogram. So far the explanation could become understood even by elementary school pupils. The following immediate question will be of course how to gather and count so many atoms. This paves the way for explaining the efforts made during the last 20 years in order to measure the number of atoms existing inside special silicon spheres. In principle, this first answer, which avoids referring to the Planck constant, is not incorrect, since this so-called silicon route for the realization of an "atomic kilogram" and a second route using a special Kibble balance for the realization of an "electric kilogram" may provide both a link between the 
Planck constant and a macroscopic mass (Stenger and Göbel, 2012).

\subsection{Higher education levels}

For a more advanced audience, possibly with university studies, or advanced pre-university studies, deserving of addressing the new definition of the kilogram based on the Planck's constant, more complex approaches are required. A friendly way of introducing the Planck constant $h$ is to motivate this particular audience with the provocative question about whether anyone knows why a fluorescent tube emits light. Next we introduce Bohr's atom model, the difference in energy $\Delta E$ between two energy levels and the emission of a photon with frequency $v$, presenting Planck's equation $\Delta E=h \nu$. Once introduced $h$, the unavoidable next question will be what the relationship of $h$ to a mass $m$ is. A possible answer is to bring to light Einstein's equation $E=m c^{2}$. Linking Einstein's equation with the mentioned Planck's relation may show the connection between $m$ and $h$. It was even presented in this way for a time on the BIPM website. Nevertheless, care should be taken when combining both equations. The mass $m$ in Einstein's equation refers to the rest mass of an object. The frequency $v$ in Planck's equation usually refers to photons emitted with that frequency. Because photons are massless particles, equating both energies to show the relationship between $h$ and $m$ requires particular consideration with respect to the mass to which $m$ refers. This mass $m$ could be, for example, the change in mass $\Delta m$ of the particle when it emits a photon of frequency $v$.

A second answer to the question of the relationship of $h$ to a mass $m$ may be given by describing the realization of an electric kilogram using the so-called Kibble balance, previously known as watt balance (Robinson and Schlamminger, 2016). Equating mechanical and electrical power, it relates the weight of $1 \mathrm{~kg}$ of mass to an electrical force needed to suspend it. The combination of two quantum effects, the quantum Hall effect discovered in 1980 by Klaus von Klitzing, in conjunction with the previously predicted Josephson effect, enables electrical power to be measured in terms of the Planck constant $h$ and frequency. According to the experience gathered in our institute of metrology over more than 20 years of teaching the SI to students with basic knowledge in natural sciences, it is not required that they necessarily have completed a course in quantum physics. The essence of both quantum effects may be explained without developing the results starting from the Schrödinger equation.

\section{Learning by doing: experimental proposals for teaching the new kilogram before the revision of the SI}

Anticipating the important difficulties that would arise in teaching the kilogram to be redefined, experimental solutions emerged already some years before its adoption. These ap- proaches assumed that incorporating hands-on learning techniques will make it easier to understand something so complex.

In 2015 two experimental educational proposals for the understanding of the kilogram definition based on fundamental constants were published: one for the electric kilogram (Chao et al., 2015) and a second for the atomic kilogram (Davis, 2015), both to $1 \%$ relative uncertainty or less. The first was a watt balance of LEGO blocks constructed at the National Institute of Standards and Technology - NIST. It allows explaining how the value of a mechanical force is precisely given by electrical measurements. Nevertheless, because the Kibble balance connects classical mechanics to quantum mechanics, addressing some equations of the quantum effects involved is inevitable. Using any version of didactic watt balances one may explain how to compensate for the mechanical power delivered by the gravitational attraction of a mass with the electrical power of a moving electromagnetic force, but not the relationship of the mass that is being weighed with the Planck constant $h$. In the own words of the authors of the LEGO balance, "Unfortunately, it still requires some abstraction to explain how electrical power is related to the Planck constant via the Josephson effect and the quantum Hall effect". The product $K_{\mathrm{J}}^{2} \times R_{\mathrm{K}}$ is needed, relating electrical voltage and resistance through the Josephson constant $K_{\mathrm{J}}=2 e / h$ and the von Klitzing constant $R_{\mathrm{K}}=h / e^{2}$.

The second is a rather simple experiment conceived by Richard Stephen Davis to explain in 2015 how the kilogram would be redefined in 2018 in terms of the Planck constant, which is closely linked to the Avogadro and atomic mass constants. After measuring the volume and mass of a highpurity aluminium cube, he estimated the number $N$ of aluminium atoms inside the cube, taking the edge dimension $a$ of the aluminium unit cell as determined in 1955 by X-ray diffraction. Next the atomic mass of aluminium $m_{\mathrm{a}}(\mathrm{Al})$ was estimated, also $m_{\mathrm{a}}\left({ }^{12} \mathrm{C}\right)$, the atomic mass of carbon-12, and the atomic mass constant $m_{u}$. As the molar mass constant $M_{u}=M\left({ }^{12} \mathrm{C}\right) / 12$ was exactly $1 \mathrm{~g} \mathrm{~mol}^{-1}$ before the revision of the SI, a value for the Avogadro constant $N_{\mathrm{A}}=M_{u} / m_{u}$ was calculated from the measurement of $m_{u}$. Using the equation for the ionization of a hydrogen-like atom, $N_{\mathrm{A}}$ and $h$ appear linked themselves and with other constants known to have very small uncertainty. Being $m_{u}=M_{u} / N_{\mathrm{A}}$ and $N_{\mathrm{A}}$ related to $h$, from the measured value of $m_{u}$ a value for $h$ was also obtained. That simple experiment allowed determining the Avogadro constant and the Planck constant, both with an uncertainty of less than $1 \%$, in close analogy to the way the most accurate experiments with silicon spheres achieved an uncertainty of a few parts in $10^{8}$.

After comparing both experiments described, we found it more interesting and less expensive to implement the experiment with the high-purity aluminium cube, as proposed by Richard Davis, than a tabletop watt balance capable of measuring gram-level masses to $1 \%$ relative uncertainty, like the 
LEGO balance quoted. With a homogenized aluminium cube (not free of imperfections and impurities) specially prepared by an Argentine manufacturer of electrolytic aluminium, the difference between the mass calculated counting atoms and the mass measured by weighing turned out to be $0.1 \%$ or less. On the other hand, the experiment with the silicon cube, adapted to engineering students, makes it possible to simultaneously implement dimensional and mass precision measurements of interest for teaching metrology at that level.

\section{Explaining the realization of the new kilogram after the revision of the SI}

Both experimental educational solutions for the realization of the kilogram mentioned in the previous section were developed before the redefinition of the SI. The purpose of such experiments changed after the redefinition. For example, in the Kibble balance approach, the experiment was initially used to measure $h$ from a mass traceable to the international prototype of the kilogram. After the redefinition, it is used to realize the definition of the kilogram from the value of $h$ fixed by the CGPM in 2018. The values of the constants to be used before the redefinition may be also different after the redefinition. In the Kibble balance approach, for example, the values of $K_{\mathrm{J}}$ and $R_{\mathrm{K}}$ used in 2015 were conventional values agreed on for electrical measurements since 1990, abbreviated as $K_{\mathrm{J}-90}$ and $R_{\mathrm{K}-90}$. Both values changed with the redefinition of the SI.

In the case of the experiment in a classroom setting with the aluminium cube described, more conceptual changes need to be taken into account. As the molar mass constant was exactly $1 \mathrm{~g} \mathrm{~mol}^{-1}$ before the revision of the SI, a value for the Avogadro constant $N_{\mathrm{A}}$ was calculated from the measurement of $m_{u}$. However, after the redefinition of the SI, the molar mass constant is no longer exactly $1 \mathrm{~g} \mathrm{~mol}^{-1}$, and $N_{\mathrm{A}}$ is now fixed in the definition of the mole.

Along with the redefinition of the units, new ways of carrying out their realizations came into effect. The so-called mises en pratique are documents indicating how the definition of an SI unit may be realized in practice. The last version of the mises en pratique for the definition of the kilogram dates from 4 August 2020 (BIPM, 2020). In the case of the silicon route for the realization of the so-called "atomic kilogram", the mass $m_{\mathrm{S}}$ of a sphere is first expressed in terms of the mass $m_{\text {atom }}$ of a single atom: $m_{\mathrm{s}}=N m_{\text {atom }}$. $N$ is the number of atoms in the sphere experimentally determined using X-ray crystal density methods. The mass of an atom expressed in kilograms is now obtained from the quotient $m_{\text {atom }} / h$. This ratio is provided to high accuracy by atom interferometry, measuring the recoil of an atom absorbing a photon (Cladé et al., 2016). Now that the numerical value of $h$ is fixed, the mass of an atom may be known with the uncertainty of the experiments determining $m_{\text {atom }} / h$ with atom interferometry. The best available results

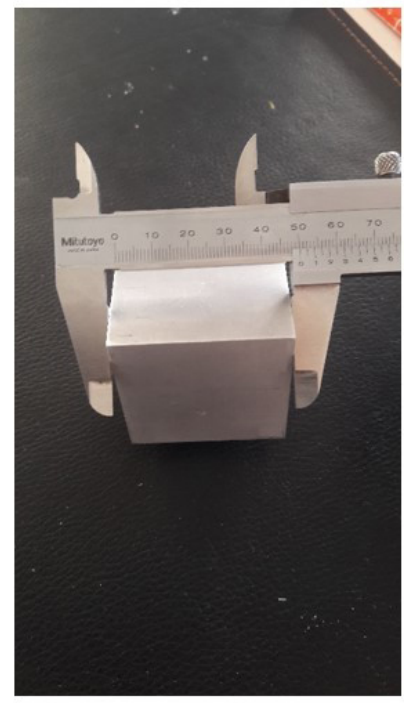

(a)

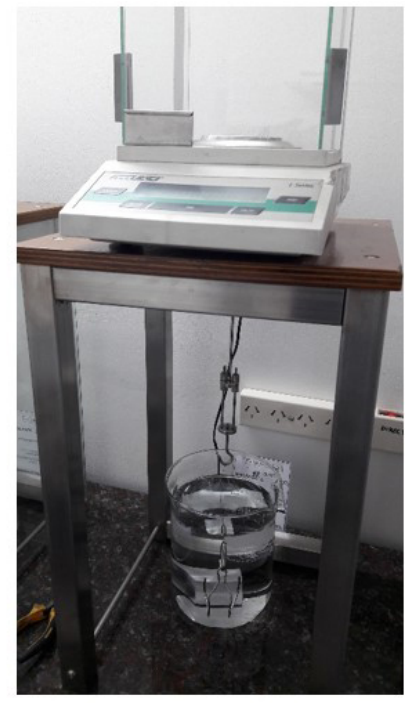

(b)
Figure 1. Experimental determination to $0.1 \%$ of the mass of an aluminium cube following the new definition of the kilogram traceable to the Planck constant. The cube volume is obtained by dimensional measurements (a), optionally also by hydrostatic weighing (b). The number of atoms $N$ is calculated taking a precise value of the edge dimension $a$ of the aluminium unit cell. The mass of the cube is then expressed as $m_{\mathrm{c}}=N m(\mathrm{Al})$, being $m(\mathrm{Al})$ the mass of a single aluminium atom, obtained from the quotient $h / m_{\text {atom }}$ provided to high accuracy by atom interferometry.

at present were reported with $2.0 \times 10^{-10}$ accuracy by Holger Müller's group at the University of California, Berkeley, in $h / m\left({ }^{133} \mathrm{Cs}\right)$ measurements (Parker et al., 2018).

If we want to use at present the didactic experiment of the aluminium cube, it should no longer be used to determine the values of certain constants which have been fixed but rather to obtain the mass of the cube following the corresponding mise en pratique of the kilogram (Fig. 1). After calculating the number of atoms $N$ it contains, we need to address a value of $h / m_{\text {atom }}$, i.e. $h / m(\mathrm{Al})$ in the case of aluminium. The simplicity of the experiment before the redefinition of the SI is no longer such, having to explain now atom interferometry, requiring knowledge of quantum physics and relativity.

\section{Conclusions}

The previous definition of the kilogram referred to an artefact that involved the risk of loss, damage, or change. The current definition of the kilogram based on a constant of nature solves the "artefact problem" and brings additional benefits. For instance, it allows one to realize mass at any scale using different technologies. Additionally, having fixed the value of the elementary charge $e$ in the definition of the ampere, the election of fixing the Planck constant $h$ in the definition of the kilogram, allowing values of $K_{\mathrm{J}}=2 e / h$ and $R_{\mathrm{K}}=h / e^{2}$ with 
zero uncertainty, brings improvements in electrical metrology. However, a complete understanding of the new definition of the kilogram will remain limited to an audience able to understand what Planck's constant is, and its relationship to the mass of a body. Teaching the new mise en pratique of the kilogram is no easy task. The realization by comparing electrical power to mechanical power requires knowledge of two quantum effects, providing the Josephson constant $K_{\mathrm{J}}$ and the von Klitzing constant $R_{\mathrm{K}}$, even using a didactic Kibble balance. The realization with the X-ray-crystal-density method (the silicon route) seems to be easier to explain. In principle, it comes from a classical idea where the mass of a pure substance can be expressed in terms of the number of elementary entities in the substance. Nevertheless, explaining that the SI value of atomic masses is obtained from atom interferometry experiments also requires advanced knowledge of physics.

Although fixing the value of the atomic mass constant $m_{u}$ would have been preferable for ease of understanding, for other reasons it was decided to fix the value of the Planck constant $h$. The new SI allows linking measurements at the atomic and quantum scales to those at the macroscopic level. The lower uncertainties now obtained for atomic mass values expressed in kilograms are still greater than those typically achieved in the comparison of atomic masses. For this reason, the atomic mass community will continue to use the non-SI unit dalton instead of the kilogram. This scenario of living with two different units of mass may change if future upgrades for atom interferometry experiments increase the accuracy by 2 orders of magnitude (Valdés, 2019). For 140 years it was quite easy to teach what $1 \mathrm{~kg}$ is. The new definition based on Planck's constant brings numerous advantages, but its understanding remains limited to a very restricted audience. Several options have been presented to explain at different levels the new definition of the kilogram, eventually using the realization based on counting atoms when the meaning of the Planck constant is not known.

Data availability. The data that support the findings of this study are available upon request to the author if required.

Competing interests. The author declares that he has no conflict of interest.

Special issue statement. This article is part of the special issue "Sensors and Measurement Science International SMSI 2020". It is a result of the Sensor and Measurement Science International, Nuremberg, Germany, 22-25 June 2020.
Acknowledgements. The author thanks Jorge Sánchez for his commitment to the hands-on teaching experiments.

Review statement. This paper was edited by Klaus-Dieter Sommer and reviewed by two anonymous referees.

\section{References}

Becker, P., De Bièvre, P., Fujii, K., Glaeser, M., Inglis, B., Luebbig, H., and Mana, G.: Considerations on future redefinitions of the kilogram, the mole and of other units, Metrologia, 44, 1-14, https://doi.org/10.1088/0026-1394/44/1/001, 2007.

BIPM: SI Brochure, 9th Edn., Appendix 2, 1-4, available at: https: //www.bipm.org/utils/en/pdf/si-mep/SI-App2-kilogram.pdf (last access: 18 January 2021), 2020.

Chao, L. S., Sclamminger, S., Newell, D. B., and Pratt, J. R.: A LEGO watt balance: An apparatus to determine a mass based on the new SI, Am. J. Phys., 83, 913-922, https://doi.org/10.1119/1.4929898, 2015.

Cladé, P., Biraben, F., Julien, L., Nez, F., and Guellati-Khelifa, S.: Precise determination of the ratio $h / m_{u}$ : a way to link microscopic mass to the new kilogram, Metrologia, 53, A75-A82, https://doi.org/10.1088/0026-1394/53/5/A75, 2016.

Davis, R. S.: What Is a Kilogram in the Revised International System of Units (SI)?, J. Chem. Educ., 92, 1604-1609, https://doi.org/10.1021/acs.jchemed.5b00285, 2015.

Fletcher, N., Davis, R. S., Stock, M., and Milton, M. J. T.: Modernizing the SI - implications of recent progress with the fundamental constants, how scientists can derive the mass of a kilogram from the fixed value of the Planck constant, available at: https://arxiv.org/ftp/arxiv/papers/1510/1510.08324.pdf (last access: 18 January 2021), 2015.

Parker, R. H., Yu, C., Zhong, W., Estey, B., and Müller, H.: Measurement of the fine-structure constant as a test of the standard model, Science, 360, 191-195, 2018.

Robinson, I. A. and Schlamminger, S.: The watt or Kibble balance: a technique for implementing the new SI definition of the unit of mass, Metrologia, 53, A46-A74, https://doi.org/10.1088/00261394/53/5/A46, 2016.

Stenger, J. and Göbel, E. O.: The silicon route to a primary realization of the new kilogram (Letter to the Editor), Metrologia, 49, L25-L27, https://doi.org/10.1088/0026-1394/49/6/L25, 2012.

Stock, M., Davis, R., de Mirandés, E., and Miltom, M. J. T.: The revision of the SI - the result of three decades of progress in metrology, Metrologia, 56, 02200, https://doi.org/10.1088/16817575/ab0013, 2019.

Valdés, J.: Reviewing the Revised International System of Units (SI), Elsevier, Adv. Imag. Elect. Phys., 211, 121-186, https://doi.org/10.1016/bs.aiep.2019.05.001, 2019. 